\title{
Acesso à saúde pelas profissionais do sexo na atenção primária: uma revisão integrativa
}

O objetivo é investigar a adesão das profissionais do sexo aos serviços de saúde da assistência primária no Brasil. Trata-se de uma revisão integrativa da literatura. Para a execução da busca dos artigos utilizou-se os seguintes descritores: prostituição, profissionais do sexo, acesso aos serviços de saúde e assistência à saúde, nas bases de dados: Literatura Latino-Americana e do Caribe em Ciências da Saúde (LILACS), Medical Literature Analysis and Retrieval System Online (MEDLINE) e U.S. National Library of Medicine (PUBMED). Os critérios de inclusão adotados foram: ano de publicação, artigos em língua portuguesa, inglesa ou espanhola e produções científicas publicadas no período de 2010 a 2019. Foram excluídos os estudos que não abordassem a temática relacionada ao objetivo da revisão. Com base no método utilizado, foram selecionados sete artigos. Os estudos expõem que as profissionais do sexo encontram dificuldades constantemente quando procuram por atendimento nos serviços públicos de atenção primária à saúde. As causas mais citadas que dificultam o acesso estão relacionadas à forma predominante a barreiras sociais e institucionais. Compreende-se que as profissionais do sexo apresentam baixa adesão aos serviços de saúde da atenção primária no Brasil. A presença de barreiras dificulta o acesso e proporciona o distanciamento dessas mulheres dos serviços público de saúde reduzindo a adesão ao sistema de atenção primária.

Palavras-chave: Prostituição; Profissionais do Sexo; Acesso aos Serviços de Saúde; Assistência à saúde.

\section{Access to health by sex professionals in primary care: an integrative review}

The objective is to investigate the adherence of sex workers to primary health care services in Brazil. This is an integrative literature review. To execute the search for the articles, the following descriptors were used: prostitution, sex workers, access to health services and health care, in the databases: Latin American and Caribbean Literature in Health Sciences (LILACS), Medical Literature Analysis and Retrieval System Online (MEDLINE) and US National Library of Medicine (PUBMED). The inclusion criteria adopted were: year of publication, articles in Portuguese, English or Spanish language and scientific productions published from 2010 to 2019. Studies that did not approached the theme related to the review objective were excluded. Based on the method used, seven articles were selected. Studies expose that sex workers constantly encounter difficulties when seeking assistance in public primary health care services. The most cited causes that hinder access are predominantly related to social and institutional barriers. It is understood that sex workers have low adherence to primary health care services in Brazil. The presence of barriers makes access difficult and provides detachment of these women from public health services, reducing the adherence to the primary care system.

Keywords: Prostitution; Sex Professionals; Access to Health Services; Health care.

Topic: Enfermagem em Saúde Pública

Reviewed anonymously in the process of blind peer.

Raissa Reis de Oliveira (iD)

Universidade de Sorocaba, Brasil

http://lattes.cnpq.br/5441520338335219

http://orcid.org/0000-0003-1443-4443

raioliveirarai@hotmail.com

Karine Honório Silva (D)

Universidade de Sorocaba, Brasil

http://lattes.cnpq.br/2803862040869220

http://orcid.org/0000-0001-6361-135X

karinesiilvah72@gmail.com

Seilla Siedler Tavares (iD

Universidade de Sorocaba, Brasil

http://orcid.org/0000-0002-3949-0102

sheilla.tavares@prof.uniso.b
Received: 04/08/2021

Approved: 03/09/2021

\author{
Márcia Féldreman Nunes Gonzaga (iD \\ Universidade de Sorocaba, Brasil \\ http://lattes.cnpq.br/3025221287848363 \\ http://orcid.org/0000-0002-8208-6914 \\ marcia.feldreman@gmail.com \\ Clayton Gonçalves de Almeida \\ Universidade de Sorocaba, Brasil \\ http://lattes.cnpq.br/6493791537446598 \\ http://orcid.org/0000-0003-2959-3965 \\ cgalmeida11@gmail.com \\ Irineu Cesar Panzeri Contini \\ Universidade de Sorocaba, Brasil \\ http://lattes.cnpq.br/3751316399780774 \\ http://orcid.org/0000-0002-7489-5527 \\ irineu.contini@prof.uniso.br
}

\section{Referencing this:}

OLIVEIRA, R. R.; SILVA, K. H.; TAVARES, S. S.; GONZAGA, M. F. N.; ALMEIDA, C. G.; CONTINI, I. C. P.. Acesso à saúde pelas profissionais do sexo na atenção primária: uma revisão integrativa. Scire Salutis, v.11, n.3, p.100-107, 2021. DOI: http://doi.org/10.6008/CBPC2236$\underline{9600.2021 .003 .0013}$ 


\section{INTRODUÇÃO}

A prostituição é definida como uma atividade que consiste em obter remuneração através de relações sexuais, sem a necessidade de associação afetiva entre a profissional do sexo e os clientes. É caracterizada por ser uma prática milenar, pois essa atividade é considerada uma das mais antigas da história e está presente na sociedade até os dias de hoje (PENHA et al., 2012). No Brasil, somente em 2002 a prostituição foi reconhecida e regulamentada como profissão pelo Ministério do Trabalho e Emprego (MTE), oficializando a atividade de profissionais do sexo como parte da Classificação Brasileira de Ocupações (CBO) (RODRIGUES, 2009; BRASIL, 2010).

Em 1984 foi implantado o Programa de Assistência Integral à Saúde da Mulher (PAISM), logo após em 2004 surgiu à proposta da Política Nacional de Atenção Integral à Saúde da Mulher (PNAISM), com o objetivo de garantir a equidade e o acesso integral a saúde para as mulheres em todas as fases da vida. Para isso, foram contempladas ações educativas, preventivas, de diagnósticos e tratamentos, além de abranger todas as outras necessidades de cada mulher de forma holística e individual (BRASIL, 2004). Porém as profissionais do sexo não têm um atendimento igualitário devido à discriminação, o que interfere na garantia dessas ações e dos seus direitos (VILLA et al., 2016).

Atualmente as profissionais do sexo ainda sofrem preconceito e discriminação pela sociedade, além disso, esse público está submetido a diversos riscos devido à vulnerabilidade da própria atividade, o que pode repercutir na situação de saúde e bem-estar dessas mulheres e ocasionar danos (FIGUEIREDO, 2010). Os problemas de saúde sexual e reprodutiva, saúde mental, uso abusivo de álcool e drogas e suscetibilidade às violências são situações prevalentes entre as profissionais do sexo, o que mostra a importância do acesso aos serviços de saúde e da assistência à saúde para essa população (BRITO et al.; 2019).

De acordo com o Ministério da Saúde (MS) (2012) Atenção Primária à Saúde (APS) “caracteriza-se por um conjunto de ações de saúde, no âmbito individual e coletivo, que abrangem a promoção e a proteção da saúde, a prevenção de agravos, o diagnóstico, o tratamento, a redução de danos e a manutenção da saúde" (BRASIL, 2012). A APS é a porta de entrada da população aos sistemas de serviços saúde, e a acessibilidade é um dos fatores essenciais para garantir a assistência, reconhecendo as necessidades de saúde de cada indivíduo a fim de reduzir a morbidade e mortalidade (LAVRAS, 2011).

$\mathrm{O}$ acesso e a cobertura universal à saúde têm como objetivo, garantir a população em níveis nacionais o acesso aos serviços de saúde de forma integral, de qualidade e sem discriminação (OPAS, 2014). Mas esse é um dos principais desafios do país, visto que milhões de pessoas não tem assistência à saúde e enfrentam dificuldades de acesso, necessitando da existência de políticas públicas para garantir a equidade em saúde, um dos princípios do Sistema Único de Saúde (SUS) para diminuir as desigualdades (VIEGAS et al., 2015).

Diante do exposto, questiona-se: Qual adesão das profissionais do sexo aos serviços de saúde da atenção primária no Brasil? Para responder esse questionamento este estudo tem como objetivo investigar a adesão das profissionais do sexo aos serviços de saúde de assistência primária no Brasil. 


\section{METODOLOGIA}

Trate-se de um estudo utilizando o método da revisão integrativa da literatura para a coleta e análise dos dados. Para tal, foram aplicadas as seguintes etapas: 1) Identificação do tema e seleção da hipótese ou questão de pesquisa; 2) Estabelecimento de critérios de inclusão e exclusão de estudos; 3) Definição das informações a serem extraídas dos estudos selecionados; 4) Avaliação dos estudos incluídos na revisão integrativa; 5) Interpretação do resultado; 6) Apresentação da revisão integrativa (MENDES et al., 2008).

Foram utilizadas as bases de dados: Literatura Latino-Americana e do Caribe em Ciências da Saúde (LILACS), Medical Literature Analysis and Retrieval System Online (MEDLINE) e U.S. National Library of Medicine (PUBMED). Para a execução da busca dos artigos, os descritores foram definidos por meio da terminologia em saúde pesquisada nos Descritores em Ciência da Saúde (DECS). Foram selecionados os descritores: prostituição; profissionais do sexo; assistência à saúde e acesso aos serviços de saúde.

Os critérios de inclusão adotados foram: ano de publicação, artigos em língua portuguesa, inglesa e espanhola e produções científicas publicadas na íntegra no período de 2010 a 2019. Foram excluídos os estudos que não abordassem a temática relacionada ao objetivo da revisão. Para seleção dos artigos, após a aplicação dos critérios de inclusão e de exclusão, foi realizada pesquisa por descritores em cada uma das bases de dados caracterizando a primeira fase da seleção. A segunda fase foi determinada por meio da leitura dos títulos e resumos e a terceira fase foi composta pela leitura dos artigos completos. Ao final da busca, foram selecionadas sete produções científicas para o desenvolvimento da revisão. Na Figura 1 encontra-se o fluxograma da busca nas bases de dados nesta revisão integrativa.

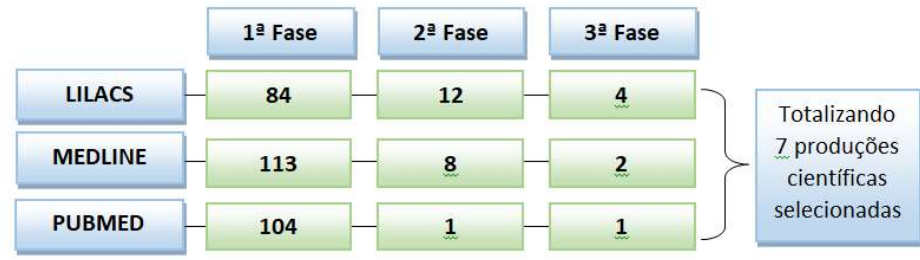

Figura 1: Fluxograma da busca dos artigos nas bases de dados.

\section{DISCUSSÃO TEÓRICA}

Com base na metodologia utilizada para realização da revisão da literatura, foram encontrados sete artigos. Desses, quatro $(57,1 \%)$ encontrados na base de dados Lilás, dois $(28,6 \%)$ na Medelín e um $(14,3 \%)$ na Pubem. Todos selecionados por meio do cruzamento dos descritores em cada base de dados. Dos estudos, seis $(85,8 \%)$ são em língua portuguesa e um $(14,3 \%)$ em língua inglesa. Com relação aos anos de publicações, o mais antigo corresponde ao ano de 2012 e o mais atual ao ano de 2019.

As produções científicas selecionadas são estudos nacionais, realizados no Brasil em diferentes estados e regiões. Dos sete artigos, quatro $(57,1 \%)$ foram desenvolvidos no Nordeste, no estado de Alagoas, Ceará, Bahia e Rio Grande do Norte, um (14,3\%) no Sudeste, em São Paulo, um (14,3\%) no Sul, no Rio Grande do Sul e um $(14,3 \%)$ representando as cinco regiões, realizado em 12 estados. Observou-se o número reduzido de produções científicas realizadas no Brasil no período de 2010 a 2019 que abordassem o tema e o objetivo do estudo. 
Os estudos foram realizados com mulheres, profissionais do sexo com faixa etária entre 18 a 55 anos. Os locais de trabalho das profissionais do sexo nos estudos analisados são bares, boates ou casas noturnas. Com a análise das produções científicas emergiram duas categorias para o resultado da revisão: adesão ao sistema primário de saúde e os motivos para procura dos serviços de saúde da atenção primária. No Quadro um pode ser observada a síntese dos artigos selecionados para o estudo, apresentados por ordem decrescente de publicação.

Quadro 1: Estudos analisados em ano decrescente com autor, local e tipo de estudo, objetivo, adesão ao sistema primário de saúde e motivo para procura ao sistema primário de saúde, Sorocaba, 2020

\begin{tabular}{|c|c|c|c|c|}
\hline Estudos & Autor / Estado/ País / Ano/ & Objetivo / Tipo de Estudo & $\begin{array}{l}\text { Adesão ao sistema primário } \\
\text { de saúde }\end{array}$ & $\begin{array}{l}\text { Motivo para procura ao } \\
\text { sistema primário de saúde }\end{array}$ \\
\hline E1 & Brito et al., 2019 & $\begin{array}{l}\text { Compreender o cotidiano de } \\
\text { trabalho e o acesso aos } \\
\text { serviços de saúde de mulheres } \\
\text { profissionais do sexo. } \\
\text { Estudo qualitativo, que utilizou } \\
\text { o método etnográfico. }\end{array}$ & $\begin{array}{l}\text { Apenas uma profissional do } \\
\text { sexo afirmou comparecer } \\
\text { frequentemente ao serviço } \\
\text { de saúde. O acesso é } \\
\text { dificultado pela presença de } \\
\text { barreiras institucionais e } \\
\text { sociais. Algumas mulheres } \\
\text { optam por serviços } \\
\text { privados. }\end{array}$ & $\begin{array}{l}\text { Buscam os serviços para } \\
\text { adquirir contraceptivos } \\
\text { (anticoncepcional e } \\
\text { preservativos), realização de } \\
\text { citologia oncótica, consultas } \\
\text { ginecológicas, e na maioria } \\
\text { dos casos quando } \\
\text { apresentam sinais e/ ou } \\
\text { sintomas (inespecíficos). }\end{array}$ \\
\hline E2 & Costa et al., 2019. & $\begin{array}{l}\text { Descrever os itinerários } \\
\text { terapêuticos e rotas críticas } \\
\text { desveladas no discurso de } \\
\text { profissionais do sexo quanto } \\
\text { ao acesso à saúde. } \\
\text { Estudo descritivo, exploratório } \\
\text { e qualitativo. }\end{array}$ & $\begin{array}{l}\text { Ocorre um distanciamento } \\
\text { dos serviços públicos } \\
\text { primários, pela demora de } \\
\text { marcação de consultas e } \\
\text { exames, além do } \\
\text { atendimento discriminatório } \\
\text { e preconceituoso. A maioria, } \\
\text { buscam por atendimento } \\
\text { periodicamente no setor } \\
\text { privado. }\end{array}$ & $\begin{array}{l}\text { Procuram pelo sistema de } \\
\text { saúde para realização de } \\
\text { citologia oncótica, consulta } \\
\text { ginecológica, realização de } \\
\text { exames laboratoriais e } \\
\text { apenas quando estão } \\
\text { doentes. }\end{array}$ \\
\hline E3 & Dourado et al., 2019 & $\begin{array}{l}\text { Investigar a não divulgação do } \\
\text { status de profissional do sexo } \\
\text { aos profissionais da saúde e a } \\
\text { sua associação com acesso e } \\
\text { utilização de serviços de } \\
\text { saúde. Estudo transversal. }\end{array}$ & $\begin{array}{l}\text { O serviço regular de } \\
\text { atendimento é a atenção } \\
\text { primária a saúde, porém } \\
\text { existe uma grande parte } \\
\text { dessas mulheres que não } \\
\text { possui adesão a nenhum } \\
\text { serviço e a presença do } \\
\text { estigma e discriminação no } \\
\text { atendimento, dificulta o } \\
\text { acesso. }\end{array}$ & $\begin{array}{l}\text { O estudo mostra apenas } \\
\text { resultados sobre a realização } \\
\text { da citologia oncótica, teste } \\
\text { de HIV, e uso de PEP. }\end{array}$ \\
\hline E4 & Paiva et al., 2013 & $\begin{array}{l}\text { Conhecer como se dá a prática } \\
\text { sexual, analisar a } \\
\text { vulnerabilidade e avaliar as } \\
\text { necessidades em saúde das } \\
\text { profissionais do sexo. } \\
\text { Estudo qualitativo de caráter } \\
\text { exploratório. }\end{array}$ & $\begin{array}{l}\text { As profissionais do sexo } \\
\text { demonstram uma adesão } \\
\text { focada na área ginecológica. } \\
\text { Percebe-se a procura por } \\
\text { serviço privado. }\end{array}$ & $\begin{array}{l}\text { As visitas às unidades de } \\
\text { saúde se resumem à } \\
\text { realização de consultas } \\
\text { médicas ginecológicas e } \\
\text { coleta da citologia oncótica. } \\
\text { Grande parte procura } \\
\text { apenas quando apresentam } \\
\text { sinais ou sintomas de } \\
\text { patologias. }\end{array}$ \\
\hline E5 & Leitão et al., 2012 & $\begin{array}{l}\text { Descrever as práticas de saúde } \\
\text { utilizadas no cotidiano de } \\
\text { profissionais do sexo. } \\
\text { Estudo qualitativo. }\end{array}$ & $\begin{array}{l}\text { Apresentam resistência pela } \\
\text { busca ao serviço público de } \\
\text { saúde, onde algumas } \\
\text { mulheres optam por } \\
\text { serviços privados, por conta } \\
\text { das dificuldades no acesso. }\end{array}$ & $\begin{array}{l}\text { O grupo se divide entre as } \\
\text { profissionais procuram o } \\
\text { sistema de saúde para } \\
\text { realizar consultas, exames } \\
\text { preventivos e de rotina, além } \\
\text { das que procuram somente } \\
\text { quando estão doentes ou } \\
\text { quando a automedicação } \\
\text { não supre suas necessidades. }\end{array}$ \\
\hline E6 & Bolzan et al., 2012 & $\begin{array}{l}\text { Observação da prática, os } \\
\text { cuidados de saúde dessas } \\
\text { mulheres e a forma que se } \\
\text { relacionam com o serviço de } \\
\text { saúde. } \\
\text { Método etnográfico }\end{array}$ & $\begin{array}{l}\text { A unidade básica de saúde é } \\
\text { o serviço mais relatado, } \\
\text { porém as mulheres } \\
\text { apresentam dificuldades } \\
\text { quanto aos horários e } \\
\text { principalmente insatisfação } \\
\text { com atendimento dos } \\
\text { profissionais da saúde } \\
\text { devido preconceito. }\end{array}$ & $\begin{array}{l}\text { Os motivos de maior } \\
\text { procurar ao sistema de } \\
\text { saúde são: Ocorrência de } \\
\text { gravidez, realização de } \\
\text { citologia oncótica e busca } \\
\text { por preservativos, além de } \\
\text { um pequeno número que } \\
\text { procura pelo dentista e por } \\
\text { exames laboratoriais. }\end{array}$ \\
\hline
\end{tabular}




\begin{tabular}{|l|l|l|l|l|}
\hline \multirow{2}{*}{ E7 } & Salmeron et al., 2012 & $\begin{array}{l}\text { Identificar o perfil } \\
\text { socioepidemiológico de } \\
\text { profissionais do sexo e } \\
\text { estabelecer medidas de } \\
\text { redução de danos. }\end{array}$ & $\begin{array}{l}\text { Algumas profissionais do } \\
\text { Resistência usual das } \\
\text { mulheres à abordagem e ao } \\
\text { acompanhamento pelos } \\
\text { serviços de saúde da } \\
\text { atenção primária. }\end{array}$ \\
\hline
\end{tabular}

Os estudos selecionados para essa revisão, expõem que as profissionais do sexo encontram dificuldades constantemente quando procuram por atendimento nos serviços públicos de atenção primária à saúde. As causas mais citadas que dificultam o acesso estão relacionadas de forma predominante ao estigma e horários limitados (BRITO et al., 2019; COSTA et al., 2019; SALMERON et al., 2012). Grande parte das profissionais do sexo que utilizam ou frequentam os serviços de saúde, buscam por atendimento na atenção primária, especificamente nas unidades básicas de saúde. Os motivos que justificam a busca pela assistência incluem consultas médicas ginecológicas, realização de exames, abertura de pré-natal ou apenas quando estão doentes (BRITO et al., 2019; COSTA et al., 2019; SALMERON et al., 2012).

Observou-se que existe uma grande insatisfação com o serviço e resistência pela procura de atendimento pelo Sistema Único de Saúde. As mulheres que apresentam maiores condições financeira desistem da assistência pela rede pública e optam por serviços privados, e as profissionais do sexo de baixa renda se afastam e deixam de frequentar os equipamentos de saúde (BRITO et al., 2019; COSTA, 2019; PAIVA et al., 2013).

Relato de maus tratos ou discriminação no atendimento prestados pelos profissionais de saúde, esteve presente em $(85,7 \%)$ dos estudos. Em três $(42,8 \%)$ dos artigos, algumas das mulheres analisadas não revelam que são profissionais do sexo (COSTA et al., 2019; PAIVA et al., 2013). A utilização pelas profissionais de sexo de programas destinado a atenção à população em situação de risco e vulnerabilidade social foi encontrada em dois (28,6\%) dos artigos analisados (LEITÃO et al., 2012; BONADIMAN et al., 2012).

A Organização Pan-Americana de saúde (2014) defende que todo indivíduo e comunidade dever ter acesso a serviços de saúde integrais, sem discriminação e de acordo com suas demandas, principalmente os grupos mais vulneráveis. Por meio da assistência integral é possível realizar a promoção e prevenção à saúde, garantindo atendimento de acordo com a carência de cada indivíduo, assegurando saúde e bemestar (OPAS, 2014).

Por meio da análise dos dados, foi possível identificar as principais barreiras que demonstram a insatisfação das profissionais do sexo com os serviços de atenção primária à saúde, como horário de funcionamento limitado, longo tempo de espera para agendamento de consultas e exames, demora no atendimento (BRITO et al., 2019; COSTA, 2019; LEITÃO et al., 2012; BONADIMAN et al., 2012), atendimento discriminatório e preconceituoso, falta de acolhimento e vínculo entre as unidades básicas e as profissionais do sexo (LEITÃO et al., 2012). A insatisfação com o atendimento prestado pelos profissionais de saúde foi relatada por grande parte das profissionais, essas expõem a falta de preparo e capacitação dos profissionais devido ao mau atendimento, com a prática de julgamentos e discriminação, onde se sentem expostas, indiferentes e humilhadas. Por esse motivo, algumas mulheres acabam não revelando sua profissão no atendimento por medo, vergonha e receio do estigma (DOURADO et al., 2019). 
A discriminação e o preconceito no atendimento prestado pelos profissionais de saúde, são um dos fatores de grande influência na adesão aos serviços de saúde. Os julgamentos e a falta de acolhimento, aumenta a resistência da procura pela assistência e inibe a possibilidade da criação de vínculo no sistema de atenção primária à saúde (ARBOIT et al., 2012). Percebe-se a necessidade da capacitação e preparo dos profissionais de saúde, visando à redução do estigma, através da prestação da assistência digna, humana e de respeito.

A presença dessas barreiras evidenciam a dificuldade de acesso e a redução pela procura da assistência (WAHED et al., 2017). Estudo aponta que a própria profissão já submete essas mulheres a diversas vulnerabilidades, como à exposição a violências, consumo de drogas, gravidez indesejada, abortos, desenvolvimento de doenças e transtornos mentais (VILLELA et al., 2015). Apesar dos riscos, a utilização dos serviços de saúde de forma preventiva é reduzida, prevalecendo a busca por cuidados apenas no processo de doença (LEITÃO et al., 2012).

Os princípios e diretrizes do Sistema Único de Saúde (SUS) são: (1) universalidade de acesso em toda e qualquer assistência; (2) integralidade, entendendo que cada indivíduo é um ser único e necessita de cuidados contínuos e integrais; (3) equidade, onde todos devem ser tratados de forma igualitária, justa e sem nenhum tipo de preconceito (BRASIL, 2000). Porém, a atual realidade do sistema público de saúde é completamente diferente das suas propostas na teoria, principalmente com relação as mulheres profissionais do sexo (DOURADO et al., 2019), que recebem atenção reduzida dos órgãos de saúde (ARBOIT et al., 2012).

A busca pelos serviços de saúde se resume em consultas ginecológicas, realização do exame de citologia oncótica, aquisição de contraceptivos, no surgimento de sinais e sintomas e uma pequena parte para realizar exames de rotina (LEITÃO et al., 2012). Um estudo realizado com profissionais do sexo, demonstrou que 19,2\% das mulheres nunca realizaram o exame de colpocitologia oncótica e $29 \%$ não frequentava nenhum tipo de serviço de saúde (DOURADO et al., 2019). A redução da adesão aos serviços da atenção primária está relacionada com os impactos das barreiras e dificuldades enfrentadas por essas mulheres (LEAL et al., 2017).

Os cuidados direcionados a área ginecológica e o foco no problema são os motivos de maior procura pelos serviços de saúde (PAIVA et al., 2015), mas a atenção primária deve conscientizar e oferecer cuidados integrais, garantindo que essas profissionais sejam assistidas através de uma assistência holística, atendendo suas necessidades de forma ampliada. Para isso, faz-se necessário a utilização de programas e ações de promoção à saúde e prevenção de doenças, tratamento e reabilitação (FONTOURA et al., 2006). A implantação do Programa de Atenção Integral à Saúde da Mulher e a Política Nacional de Atenção Integral à Saúde da Mulher, surgiu visando garantir as necessidades de saúde e ampliar o acesso integral à saúde de todas as mulheres (BRASIL, 2004), mas as profissionais do sexo acabam sendo menosprezadas perante a regulamentação de estratégias. Por terem seus direitos violados, a vulnerabilidade aumenta, necessitando de ajustes e fortalecimento das políticas públicas voltada para esse público a fim de garantir todos os seus direitos e uma assistência à saúde de qualidade como qualquer outro cidadão (ARBOIT et al., 2012). 


\section{CONCLUSÕES}

Após análise das produções científicas, compreende-se que as profissionais do sexo apresentam baixa adesão aos serviços de saúde da atenção primária no Brasil. A presença de barreiras, dificulta o acesso e proporciona o distanciamento dessas mulheres dos serviços público de saúde, reduzindo a adesão ao sistema de atenção primária. O estigma no atendimento e o horário de funcionamento limitado são os obstáculos determinantes de maior influência no acesso e na busca por assistência.

A procura por uma assistência integral e contínua ainda é mínima, predomina-se a utilização dos serviços de saúde da atenção primária com foco no problema, no surgimento de queixas e sinais e sintomas, desenvolvimento de doenças, e em alguns casos para cuidados específicos e direcionados a ginecologia, demonstrando resistência pela procura por atendimento de forma preventiva. Em razão dos desafios e dificuldades enfrentadas pelas profissionais do sexo nos serviços públicos do Brasil, evidencia-se a preferência pela rede privada, sistema capaz de atender as demandas e suprir suas necessidades quando há condições financeiras suficiente para ter acesso a essas instituições, mas essa não é a realidade da maioria dessas mulheres devido as condições socioeconômicas reduzida, e por esse motivo acabam desassistidas dos sistemas de saúde.

Diante a limitação de produções científicas que abordassem o tema e objetivo da revisão, faz-se necessário a ampliação dos estudos e discussões sobre a temática, para que estratégias sejam estabelecidas a fim de reduzir as dificuldades e ampliar o acesso, permitindo que as profissionais tenham seus direitos garantidos, suas necessidades atendidas e procurem os equipamentos de saúde com maior frequência, aumentando assim, a adesão desse grupo a atenção primária.

\section{REFERÊNCIAS}

ARBOIT, J.; HIRT, M. C.; GEHLEN, R. G. S.; BORTOLI, V. S.; COSTA, M. C.; SILVA, E. B.. Situações de vulnerabilidade à violência de mulheres profissionais do sexo: interfaces no campo da saúde. Rev. Enferm. UFPE, v.8, n.2, 2014. DOI: https://doi.org/10.5205/reuol.4597-376831ED.0810supl201422

BONADIMAN, P. O. B.; MACHADO, P. S.; LOPEZ, L. C.. Práticas de saúde entre prostitutas de segmentos populares da cidade de Santa Maria-RS: o cuidado em rede. Physis, Rio de Janeiro, v.22, n.2, p.779-801, 2012. DOI:

https://doi.org/10.1590/S0103-73312012000200020

BRITO, N. S.; BELÉM, J. M.; OLIVEIRA, T. M.; ALBUQUERQUE A. G.; QUIRINO, G. S.. Cotidiano de trabalho e acesso aos serviços de saúde de mulheres profissionais do sexo. Rev. Rene, v.20, n.1, p.1-9, 2019.

COSTA, A. S. S. F.; SOUZA, M. N. L. A.; SOUZA, A. R.; LIMA, A S. C.; OLIVEIRA, M. T.. Passos CR. Itinerários terapêuticos e rotas críticas de profissionais do sexo no acesso aos serviços de saúde. Revisa, v.9, n.1, p.53-64, 2019.

DOURADO, I.; GUIMARÃES, M. D. C.; DAMACENA, G. N.; MAGNO, L.; SOUZA JÚNIOR, P. R. B.; SZWARCWALD, C. L.; BRAZILIAN, F. S. W.. Sex work stigma and non-disclosure to health care providers: data from a large RDS study among FSW in Brazil. BMC Int Health Hum Rights, v.5, n.19, p.1-8.

\section{DOI: $\underline{\text { https://doi.org/10.1186/s12914-019-0193-7 }}$}

FIGUEIREDO, R.; PEIXOTO, M.. Profissionais do sexo e vulnerabilidade. BIS, Bol. Inst. Saúde, v.12, n.2, 2010.

FONTOURA, R. T.; MAYER, C. N.. Uma breve reflexão sobre a integralidade. Rev. Bras. Enferm., Brasília, v.59, n.4, p.532536, 2006.

LAVRAS, C.. Atenção primária à saúde e a organização de redes regionais de atenção à saúde no Brasil. Saude Soc., São Paulo, v.20, n.4, p.867-874, 2011.

LEAL, C. B. M.; SOUZA, D. A.; RIOS, M. A.. Aspectos de vida e saúde das profissionais do sexo. Rev. Enferm. UFPE, v.11, n.11, 2017.

LEITÃO, E. F.; COSTA, L. L. S.; BRÊDA, M. Z.; ALBUQUERQUE M, C, D.; JORGE, J. S.. A prática cotidiana de saúde das profissionais do sexo. Rev. Bras. Promoç. Saúde, v.25, n.3, p.294-304, 2012. DOI: https://doi.org/10.5020/18061230.2012.2259

MENDES, K. D. S.; SILVEIRA, R. C. C. P.; GALVAO, C. M. Revisão integrativa: método de pesquisa para a incorporação de evidências na saúde e na enfermagem. Texto Contexto Enferm., Florianópolis, v.17, n.4, p.758-764, 2008. 
BRASIL. Política nacional de atenção básica. Brasília: Ministério da Saúde, 2012.

BRASIL. Política nacional de atenção integral à saúde da mulher: princípios e diretrizes. Brasília: Ministério da Saúde, 2004.

BRASIL. Sistema Único de Saúde: Princípios e Conquistas. Brasília: Ministério da Saúde, 2000.

MTE. Ministério do Trabalho e Emprego. Classificação Brasileira de Ocupações. Brasília: Ministério do Trabalho e Emprego, 2010.

OPAS. Organização Pan-Americana da Saúde. Plano Estratégico da Organização Pan-Americana da Saúde. Estados Unidos: OPAS, 2014.

PAIVA, L. L.. A vivência das profissionais do sexo. Saúde Debate, Rio de Janeiro, v.37, n.98, p.467-476, 2013.

PENHA, J. C.; CAVALCANTI, S. D. C.; CARVALHO, S. B.; AQUINO, P. S.; GALIZA, D. D. F.; PINHEIRO, A. K. B.. Caracterização da violência física sofrida por prostitutas do interior piauiense. Rev. Bras. Enferm., v.28, n.6, p.984-900, 2014. DOI: http://dx.doi.org/10.1590/S0034$\underline{71672012000600015}$

RODRIGUES, M. T.. A prostituição no Brasil contemporâneo: um trabalho como outro qualquer?. Rev. Katálysis,
Florianópolis, v.12, n.1, p.68-76, 2009. DOI:

http://dx.doi.org/10.1590/S1414-49802009000100009

SALMERON, N. A.; PESSOA, T. A. M.. Profissionais do sexo: perfil socioepidemiológico e medidas de redução de danos. Acta Paul. Enferm., São Paulo, v.25, n.4, p.549-554, 2012. DOI: http://dx.doi.org/10.1590/S0103-21002012000400011

VIEGAS, A. P. B.; CARMO, R. F.; LUZ, Z. M. P.. Fatores que influenciam o acesso aos serviços de saúde na visão de profissionais e usuários de uma unidade básica de referência. Saude Soc., São Paulo, v.24, n.1, p.100-112, 2015. DOI: http://dx.doi.org/10.1590/S010412902015000100008

ILLELA, W. V.; MONTEIRO, S.. Gênero, estigma e saúde: reflexões a partir da prostituição, do aborto e do HIV/aids entre mulheres. Epidemiol. Serv. Saúde, Brasília, v.24, n.3 p.531-540, 2015. DOI: https://doi.org/10.5123/S167949742015000300019

VILLA, E. A.; CÂNDIDO, M. C. R. M.; SISTE, L. F.. A assistência das profissionais do sexo no Brasil: uma revisão integrativa. Nurs Health, v.1, n.1, p.92-102, 2016.

WAHED, T.; ALAM, A.; SULTANA, A.; RAHMAN, M.; ALAM, N.; MARTENS, M.. Barriers to sexual and reproductive healthcare services as experienced by female sex workers and service providers in Dhaka city. PLOS ONE, v.12, n.7, p.119, 2017. DOI:

https://doi.org/10.1371/journal.pone.0182249

A CBPC - Companhia Brasileira de Produção Científica (CNPJ: 11.221.422/0001-03) detém os direitos materiais desta publicação. Os direitos referem-se à publicação do trabalho em qualquer parte do mundo, incluindo os direitos às renovações, expansões e disseminações da contribuição, bem como outros direitos subsidiários. Todos os trabalhos publicados eletronicamente poderão posteriormente ser publicados em coletâneas impressas sob coordenação da Sustenere Publishing, da Companhia Brasileira de Produção Científica e seus parceiros autorizados. Os (as) autores (as) preservam os direitos autorais, mas não têm permissão para a publicação da contribuição em outro meio, impresso ou digital, em português ou em tradução. 